\title{
Thymus-expressed chemokine secreted by breast cancer cells promotes metastasis and inhibits apoptosis
}

\author{
LU CHEN $^{1}$, SHUMING ZHANG ${ }^{2}$, YAQIAN SHEN ${ }^{1}$, LINZENG QI $^{1}$, \\ ZHAOLIN ZHANG $^{3}$, HUA TIAN ${ }^{1}$ and ZHIGENG ZOU ${ }^{4}$
}

\begin{abstract}
${ }^{1}$ Department of Anatomy, Histology and Embryology, School of Basic Medical Science, Shandong University, Jinan, Shandong 250012; ${ }^{2}$ Department of Radiotherapy, Shandong Provincial Qianfoshan Hospital, The First Hospital Affiliated with Shandong First Medical University, Jinan, Shandong 250014; ${ }^{3}$ Department of Special Examination, Penglai People's Hospital, Penglai, Shandong 265600; ${ }^{4}$ Department of Oncology, Shandong Provincial Hospital

Affiliated to Shandong University, Jinan, Shandong 250021, P.R. China
\end{abstract}

Received November 1, 2019; Accepted March 10, 2020

DOI: 10.3892/or.2020.7575

\begin{abstract}
The aim of the present study was to investigate the underlying mechanisms of thymus-expressed chemokine (TECK) autocrine signaling, and its effect on carcinogenesis and the development of breast cancer. The present study also assessed epithelial-mensenchymal transition (EMT) and cell migration, invasion, proliferation and apoptosis. Breast cancer cell lines MCF-7 and MDA-MB-231 were used in the present study, and TECK basic expression in cancer cells was investigated using western blotting (WB). EMT markers, Akt pathway molecules and apoptosis indicators were detected by reverse transcription-quantitative PCR or WB. In order to assess migration and invasion, wound healing and Matrigel invasion assays were performed. Moreover, flow cytometry was used to assess the rate of proliferation and apoptosis. In vivo experiments were conducted in nude mice to assess cancer growth. It was revealed that breast cancer cells could secrete TECK in an autocrine manner. Furthermore, TECK could increase cell migration and invasion by promoting EMT and inhibit apoptosis via the Akt signaling pathway.
\end{abstract}

\section{Introduction}

Breast cancer is the common type of cancer in females, and its morbidity rates have been increasing since the 1970s (1). Moreover, the health burden of breast cancer is increasing in China, with $>1.6$ million individuals being diagnosed and 1.2 million mortalities each year. Breast cancer is the most common type of cancer in Chinese female patients; cases

Correspondence to: Dr Zhigeng Zou, Department of Oncology, Shandong Provincial Hospital Affiliated to Shandong University, 324 Jingwu Weiqi Road, Jinan, Shandong 250021, P.R. China

E-mail: zou_zhigeng@163.com

Key words: thymus-expressed chemokine, autocrine, epithelialmesenchymal transition, apoptosis, breast cancer in China account for $12.2 \%$ of all newly diagnosed cancers and $9.6 \%$ of all mortalities from breast cancer worldwide (2). Furthermore, in America, 231,840 new cases of invasive breast cancer and 40,290 breast cancer mortalities were estimated to occur among women in 2015 (3). In addition, from 2012-2016, the breast cancer incidence rate increased by $\sim 0.3 \%$ per year (4). Therefore, it is important to investigate the etiological factors and the related factors that may accelerate the progression of breast cancer.

It has been revealed that the immune system plays an important role in carcinogenesis and cancer progression. Chemokines, which are immune substances, can act as cancer promoters in tumor development (5), but also have an antitumor immune response function (6). Therefore, it is important to investigate dual-directional regulation substances, such as chemokines. Thymus-expressed chemokine (TECK), also known as CCL25, is an important chemokine. Its receptor is CCR9.

TECK is mainly expressed in the thymus and small intestine (7), however it can also be secreted from the spleen and pancreatic stellate cells (8). Li et al (9) revealed that TECK could be secreted from endometrial stromal cells and may mediate regulatory T-cell differentiation. Moreover, TECK plays a role in the development of T cells. TECK is a chemotactic factor that acts on thymocytes, macrophages and dendritic cells, and results in the recruitment of thymic precursors, migration of thymocytes within the thymus and recruitment of thymic dendritic cells (10).

Previous studies revealed that TECK is associated with survival rate (11) and drug-resistance in breast cancer (12). But as a chemokine expressed by the thymus, the role of TECK on carcinogenesis and progression of breast cancer is not fully understood. Furthermore, whether breast cancer cells can secrete TECK requires further investigation.

The aims of the present study were to investigate the role and underlying mechanism of TECK on cell migration, invasion, epithelial-mesenchymal transition, proliferation and apoptosis in breast cancer. Furthermore, the effect of TECK on carcinogenesis and progression in breast cancer was examined, which may facilitate the development of novel therapeutic strategies for breast cancer treatment. 


\section{Materials and methods}

Cell culture and reagents. The two human breast cancer cell lines MCF-7 and MDA-MB-231 were obtained from the American Type Culture Collection. MCF-7 and MDA-MB-231 cells were cultured in RPMI-1640 medium (Hyclone; GE Healthcare Life Sciences) with 10\% FBS (Gibco; Thermo Fisher Scientific, Inc.) in $5 \% \mathrm{CO}_{2}$ at $37^{\circ} \mathrm{C}$. Both cell lines were stimulated with TECK (PeproTech, Inc.) at 0, 25, 50, $100 \mathrm{ng} / \mathrm{ml}$ to assess the effect of TECK on the expression of epithelial-mensenchymal transition (EMT) biomarkers, and cell migration and invasion. LY294002 (Cell Signaling Technology, Inc.) was used to inhibit the phosphorylation of Akt.

Immunohistochemistry. This study was approved by the Ethics Committees of Shandong Provincial Hospital (NSFC: approval no. 2019-201). Thymus tissues from three healthy volunteers (37, 55 and 61 years old) were collected from Shandong University Qilu Hospital from June to July 2019, and written informed consents were obtained prior to sample collection. Tissues were embedded with paraffin and cut into $5 \mu \mathrm{m}$-thick sections. Tissue sections were dewaxed in xylene and rehydrated in graded alcohol concentrations. Sodium citrate buffer was used for antigen retrieval. The endogenous peroxidase activity of tissues was blocked, and tissues were then incubated with the primary antibody anti-TECK $(1: 1,000$; product code ab200343) overnight at $4^{\circ} \mathrm{C}$ : and the secondary antibody anti-rabbit $(1: 1,000$; product code ab97080; both from Abcam). DAB was used to reveal the area targeted by the primary antibodies, and nuclei were counterstained with hematoxylin.

Interference and enhance of gene expression. MCF-7 and MDA-MB-231 cells were seeded $\left(1 \times 10^{5}\right)$ in a 6-well plate (NEST Biotechnology) and cultured overnight. Cells were transfected with TECK overexpression, silencing and negative control vectors, which contained green fluorescent protein (GFP) gene. Viral vectors Ubi-MCS-3FLAG-CBh-gcGFP-IRES-puromycin for overexpression and hU6-MCS-CBH-gcGFP-IRES-puromycin for silencing were used. The sequences for TECK silencing were as follows: Small interfering RNA (si)TECK1, 3'-GCU CCUGGAUGCUCGAAAUTT-5'; siTECK2, 3'-CCAAGU UUAGCAAUCCCAUTT-5'; and SiTECK3, 3'-CCUCCUGAU AUCAGCUAAUTT-5'. The sequence for CCR9 silencing was: 3'-GCUUGA AGCUGUCGUCUAUTT-5'. Culture medium was changed after $12 \mathrm{~h}$, and then puromycin was added into the culture medium in order to select cells transfected with vectors. The transfection efficiency was assessed by fluorescence microscope, reverse transcription-quantitative PCR (RT-qPCR) and western blotting.

Western blotting. Cells were lysed by RIPA buffer (Beyotime Institute of Biotechnology) with protease inhibitor cocktail (20X), ultrasonication was performed and then cells were centrifuged at $12,000 \times \mathrm{g}$ at $4^{\circ} \mathrm{C}$ for $30 \mathrm{~min}$. The supernatant was collected and the concentration of protein was determined using the BCA Protein Assay kit (Thermo Fisher Scientific), and then, loading buffer (5X) was added. The sample was separated with $10 \%$ SDS-PAGE gel (40 $\mu$ g protein per lane) by electrophoresis. Then, proteins were electrotransferred onto a PVDF membrane (Invitrogen; Thermo Fisher Scientific, Inc.). The membrane was blocked with 5\% BSA (Beijing Solarbio Science \& Technology Co., Ltd.), and incubated at $4^{\circ} \mathrm{C}$ overnight with the primary antibodies: Akt $(1: 1,000$; product no. 4691), and phosphorylated (p)-Akt (1:1,000; product no. 4060; Cell Signaling Technology, Inc.), TECK (1:250; product code ab400343; Abcam), E-cadherin (1:1,000; product no. 3195), vimentin (1:1,000; product no. 5741), Snail (1:250; product no. 3879), $\beta$-actin (1:1,000; product no. 4970), cleaved caspase-3 (1:500; product no. 9664) and Bax (1:500; product no. 5203; all from Cell Signaling Technology, Inc.). Membranes were washed with TBST three times (10 min per time),incubated with horseradish peroxidase-conjugated Rabbit secondary antibodies (1:10,000; cat. no. ZB-2301; Zhongshan Golden Bridge Biotechnology; OriGene Technologies), and then washed thrice with TBST. Protein bands were visualized with an electrochemiluminescence reagent (Advansta, Inc.) and the software ImageJ (version 1.48; National Institutes of Health) was used for densitometry.

$R T$ - $q P C R$. Total RNA was isolated using TRIzol ${ }^{\circledR}$ (Invitrogen; Thermo Fisher Scientific, Inc.) according to the manufacturer's instructions. cDNA was reverse transcribed from the mRNA using Revert Aid First Strand cDNA Synthesis kit (Thermo Fisher Scientific, Inc.) following the manufacturer's protocols. mRNAs of GAPDH and TECK, and the EMT biomarkers E-cadherin, vimentin and Slug were detected by RT-qPCR using Maxima SYBR Green qPCR Master mix (Thermo Fisher Scientific, Inc.). Data were obtained and analyzed using the Mx4000 Multiplex qPCR System (Stratagene; Agilent Technologies, Inc.) equipped with version 3.0 software. RT-qPCR reaction conditions were as follows: Initial denaturation at $95^{\circ} \mathrm{C}$ for $30 \mathrm{sec}$, followed by 40 cycles at $95^{\circ} \mathrm{C}$ for $5 \mathrm{sec}$ and $60^{\circ} \mathrm{C}$ for $34 \mathrm{sec}$. The $2^{-\Delta \Delta \mathrm{Cq}}$ method was utilized for the quantification of gene expression, with GAPDH as an endogenous control (13). The primers used were as follows: E-cadherin forward, 5'-CTGATGCTGATGCCCCCAATA-3' and reverse, 5'-CAGTTTCTGCATCTTGCCAGG-3'; Snail forward, 5'-AGGCAGCTATTTCAGCCTCC-3' and reverse, 5'-CACATCGGTCAGACCAGAGC-3'; TECK forward, 5'-TTTGAAGACTGCTGCCTGG-3' and reverse, 5'-GTCTTC TTCCTAACAAGCC-3'; and GAPDH forward, 5'-GCCGCA TCTTCTTTTGCGTCGC-3' and reverse, 5'-TCCCGTTCT CAGCCTTGACGG-3'.

Wound-healing assay. Human breast cells were cultured in 6-well dishes with RPMI-1640 medium (Hyclone; GE Healthcare Life Sciences) with 10\% FBS (Gibco; Thermo Fisher Scientific, Inc.). When the cells grew to $>90 \%$ confluency, a scratch was performed with a $200 \mu$ l pipette tip to make a linear wound in the central area. Then cells were washed with PBS, and migrated cells were counted under a phase-contrast microscope (magnification x100) after 24 and $48 \mathrm{~h}$.

Matrigel invasion assay. Matrigel (BD Biosciences) was thawed at $4^{\circ} \mathrm{C}$ overnight and diluted in RPMI-1640 cell culture media. Then, $60 \mu 1$ diluted Matrigel was added into the upper chamber of a 24-well Transwell plate (Corning, 
Inc.) and incubated at $37^{\circ} \mathrm{C}$ for $30 \mathrm{~min}$. Cells were seeded $\left(5 \times 10^{4}\right)$ on the Matrigel in the upper chamber with DMEM, which was supplemented with $1 \% \mathrm{FBS}$, and the lower chamber was coated with RPMI-1640 medium with $10 \%$ FBS. Non-invaded cells were removed with a cotton swab. The invaded cells, which adhered to the lower surface of the filter, were fixed with methanol at room temperature for $30 \mathrm{~min}$ and stained with $0.1 \%$ crystal violet (Beijing Solarbio Science \& Technology Co., Ltd.) at room temperature for $20 \mathrm{~min}$. The number of invaded cells in five random fields (magnification $\mathrm{x} 100$ ) on the underside of the filter was counted under a light microscope (14).

Cell Counting Kit-8 (CCK-8) assay. Cells (5,000) with $100 \mu 1$ medium were seeded in 96-well dishes and cultured for $48 \mathrm{~h}$ under different stimulations. Medium in each well was replaced with $100 \mu \mathrm{l}$ serum-free medium containing $10 \mu \mathrm{l}$ CCK-8 reagent and cultured for $2 \mathrm{~h}$. The absorbance was measured at $450 \mathrm{~nm}$ using a microplate reader.

Annexin V/PI assay. Cells were seeded (0.4 million in 6-well dishes. After $12 \mathrm{~h}$, cells were treated with the blank, $100 \mathrm{ng} / \mathrm{ml}$ TECK, LY294002 +100 ng/ml TECK and LY294002 for $24 \mathrm{~h}$. Cells were then treated with $750 \mu \mathrm{M} \mathrm{H}_{2} \mathrm{O}_{2}$ for $12 \mathrm{~h}$ in order to induce apoptosis. Cells were collected and stained according to the manufacturer's protocol. The ratio of apoptotic cells was detected with flow cytometry (BD Biosciences) and the data were analyzed using FlowJo software (version 7.6; BD Biosciences).

Hoechest 33342 staining. Cells were treated as aforementioned in the 'Annexin V/PI assay' section. Hoechest solution 33342 was purchased from Beyotime Institute of Biotechnology. Staining solution was added into wells to cover cells for $5 \mathrm{~min}$. The staining solution was then removed and cells were washed thrice with PBS before observation under a fluorescence microscope. The apoptotic cells in five random fields were counted with at a magnification of $\mathrm{x} 100$.

Cell cycle detection. Cells were seeded (0.1 million) in 6-well plates. The treatment was the same as aforementioned in the CCK-8 assay. PI was used for DNA staining. The fluorescence signals were detected by flow cytometry and data were processed using ModFit (Windows Version 3.2; Verity Software House).

Nude mice xenograft model. Forty-eight female nude mice (4 weeks old) were purchased from Model Animal Research Center of Nanjing University and housed four animals per cage under standard conditions $\left(24^{\circ} \mathrm{C} \pm 2^{\circ} \mathrm{C}, 50 \pm 10 \%\right.$ relative humidity, $12 \mathrm{~h}$ light/dark cycles) and with unlimited access to standard rodent maintenance feed (KEAO XIELI FEED Co., Ltd.) and water. Hygienic conditions were maintained by weekly cage changes. Animal health and behavior were monitored every day and body weights were assessed weekly over the course of the study. The animals were divided into 6 groups (MCF-7: TECK-OE, NC and siTECK; MDA-MB-231: TECK-OE, NC and siTECK) with 8 animals in each group. Newly digested cells $\left(2 \times 10^{6}\right)$ were injected to the oxter region of the animal. The tumors were measured every other day and the tumor volume was estimated according to the following formula: Volume=length $\mathrm{x}$ width $^{2} \mathrm{x} 0.52$. All animals were sacrificed by overdose ( $>120 \mathrm{mg} / \mathrm{kg}$ body weight) intraperitoneal injection of pentobarbital after 40 days. The death was verified by loss of spontaneous breathing. All procedures were approved by The Use Committee for Animal Care of Shandong University.

Statistical analysis. Data from experiments are presented as the mean $\pm \mathrm{SD}$. The comparisons among different groups (>2 groups) were analyzed by one-way analysis of variance (one-way ANOVA) with a Tukey's post hoc test, while Student's t-test was used for comparisons between 2 groups. SPSS 22.0 (IBM Corp.) was used for statistical analysis.

\section{Results}

TECK is secreted by breast cancer cells in an autocrine manner, and is highly expressed in adult atrophic thymus tissue. Western blotting was used to detect the protein expression of TECK in breast cancer cells, and IHC was used to detect TECK expression in thymus tissue. It was revealed that adult atrophic thymus tissue had high TECK expression (Fig. 1A). Furthermore, it was demonstrated that breast cancer cells could synthesize and secrete TECK (Fig. 1B). Based on analysis of The Cancer Genome Atlas (TCGA) database, the present results indicated that primary breast cancer tissues have a high TECK expression compared with healthy breast tissues (Fig. 1C; $\mathrm{P}=0.0067$ ).

TECK promotes cell migration and invasion via the Akt signaling pathway. The present results indicated that TECK significantly promoted cell migration in breast cancer (Fig. 2A and B). Furthermore, the percentage of migrated cells in the TECK treatment group was increased compared with the control group, and the effect could be suppressed by blocking the phosphorylation of Akt using LY294002 (TECK group vs. LY294002+TECK group) (Fig. 2C). Thus, TECK may promote cell migration via the Akt signaling pathway in breast cancer. The present results also indicated that TECK treatment promoted cell invasion compared to the control group, and similarly, this effect could also be suppressed by blocking the Akt signaling pathway (TECK group vs. LY294002+TECK group) (Fig. 2D and E).

TECK promotes the EMT process in a dose-dependent manner. To investigate whether TECK could affect the process of EMT in breast cancer, MCF-7 and MBA-MD-231 cells were treated with TECK $(0,25,50$ and $100 \mathrm{ng} / \mathrm{ml})$ for $48 \mathrm{~h}$. Then, the expression levels of the EMT biomarkers E-cadherin, vimentin and Snail were detected by western blotting. It was revealed that there was a dose-dependent relationship between TECK treatment and the EMT process, as, EMT was enhanced as the dose of TECK increased. Moreover, this effect was demonstrated by the decreased protein expression of E-cadherin, and the increased expression levels of vimentin and Snail (Fig. 3A and B).

TECK promotes the EMT process via the Akt pathway. In order to further examine the possible signal molecule underlying the 

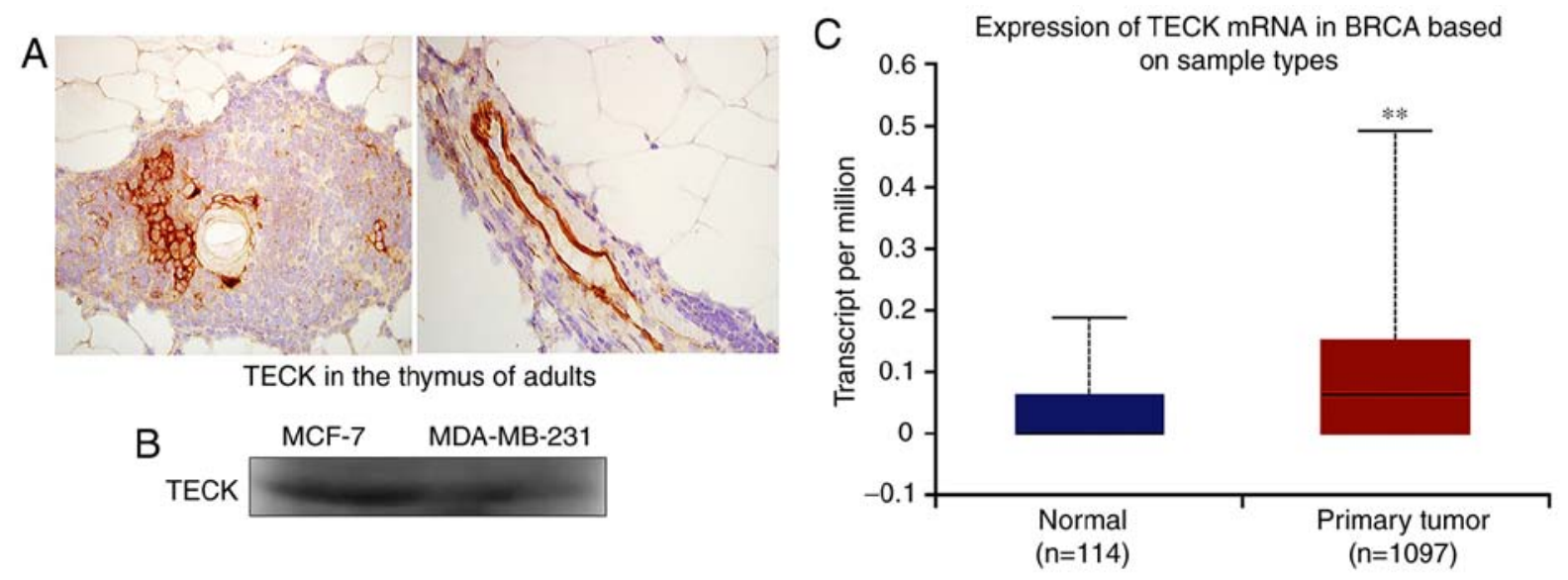

Figure 1. TECK is expressed in the adult thymus and breast cancer cells, and TECK mRNA expression is higher in primary tumor tissues compared with healthy tissues. (A) TECK expression in adult atrophic thymus tissues. (B) TECK protein expression in MCF-7 and MDA-MB-231 cells. (C) TECK mRNA expression in primary breast tumor tissues compared with healthy breast tissues, based on TCGA database. ${ }^{* *} \mathrm{P}<0.01$. TECK, thymus-expressed chemokine.

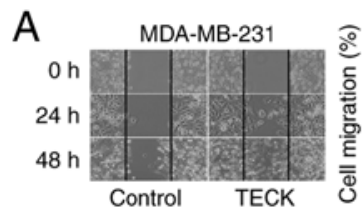

C

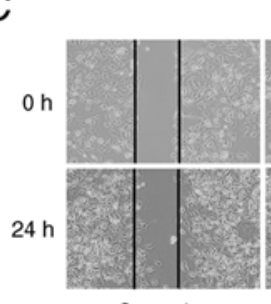

Control
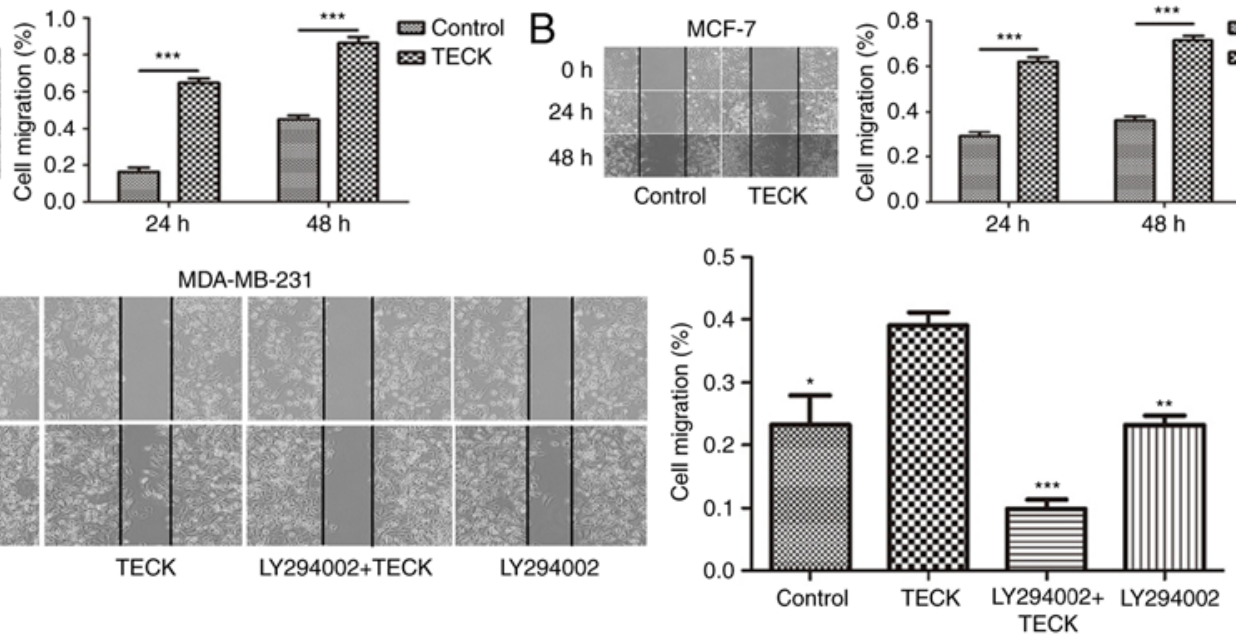
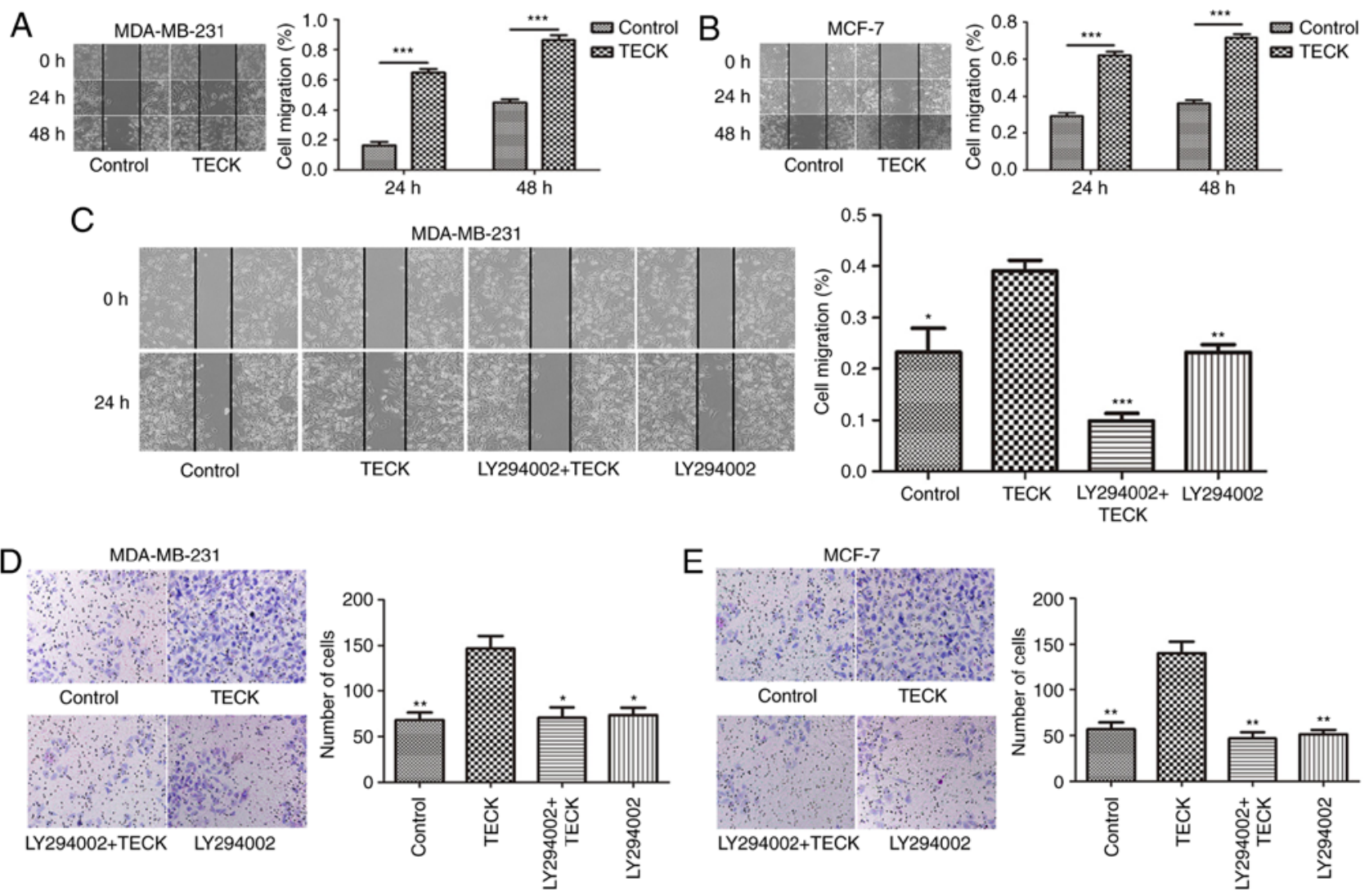

Figure 2. TECK promotes cell migration and invasion via the Akt signaling pathway in breast cancer cells. (A) MDA-MB-231 and (B) MCF-7 were treated with TECK for 24 and $48 \mathrm{~h}$, and then a wound-healing assay was used to detect migration capacity. ${ }^{* * *} \mathrm{P}<0.001$. (C) Cells were treated with the Akt inhibitor LY294002 prior to TECK stimulation. Capacity of migration was assessed by wound healing assay after $24 \mathrm{~h}$ stimulation with TECK. Magnification, x100. ${ }^{*} \mathrm{P}<0.05,{ }^{* *} \mathrm{P}<0.01,{ }^{* * *} \mathrm{P}<0.001$ compared with the TECK group. (D) MDA-MB-231 and (E) MCF-7 were treated with TECK for 24 and $48 \mathrm{~h}$ in a Transwell assay with Matrigel. After $48 \mathrm{~h}$, the cells were stained with crystal violet. Magnification $\mathrm{x} 100 .{ }^{*} \mathrm{P}<0.05$, ${ }^{* *} \mathrm{P}<0.01 \mathrm{compared}$ with the TECK group. TECK, thymus-expressed chemokine.

effect of TECK on EMT, the Akt phosphorylation inhibitor LY294002 was used to block signal transduction. Moreover, the biomarkers of EMT Akt and p-Akt were detected by western blotting. It was revealed that $100 \mathrm{ng} / \mathrm{ml}$ TECK treatment could significantly increase the level of EMT compared to the control group, and the promotion could be suppressed by Akt phosphorylation inhibitor LY294002. Therefore, the present results indicated that TECK promoted the EMT process via the Akt signaling pathway (Fig. 3C and D). Furthermore, similar results were obtained from the RT-qPCR results, which detected the mRNA expression levels of EMT markers. As revealed in Fig. 3E and F, EMT was promoted by TECK treatment (TECK group vs. control group), which could be suppressed by CCR9 interference (siCCR9+TECK 

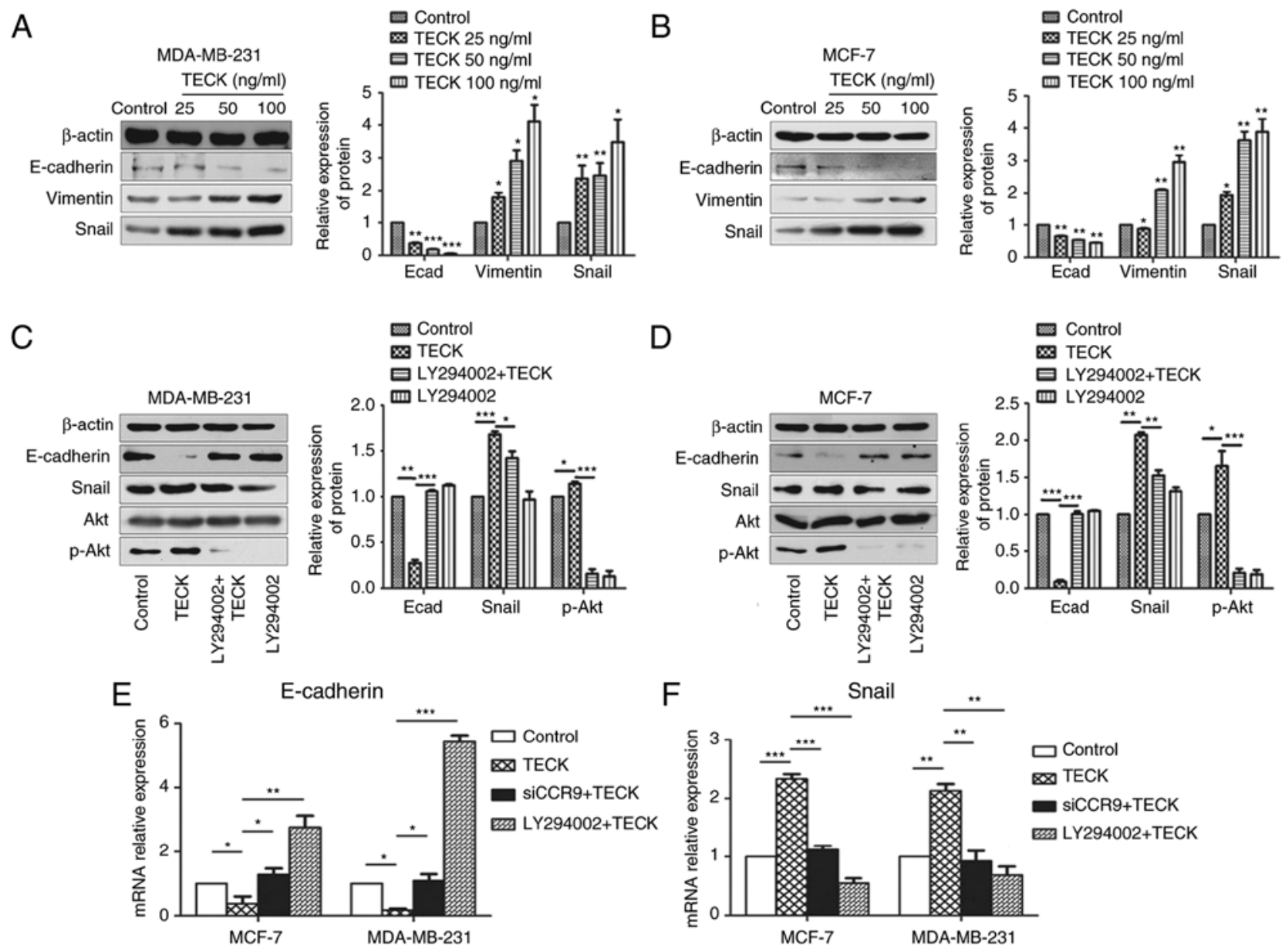

Figure 3. TECK promotes EMT mediated by the Akt signaling pathway in a dose-dependent manner in breast cancer cells. (A) MDA-MB-231 and (B) MCF-7 were treated with TECK 0,25,50 and $100 \mathrm{ng} / \mathrm{ml}$, and then the expression levels of E-cadherin, vimentin and Snail were detected by western blotting. ImageJ was used for densitometry. ${ }^{*} \mathrm{P}<0.05,{ }^{* *} \mathrm{P}<0.01,{ }^{* * *} \mathrm{P}<0.001$ compared with the control group. (C) MDA-MB-231 and (D) MCF-7 cells were treated with the Akt inhibitor LY294002 before adding TECK. The expression levels of E-cadherin, Snail, Akt and p-Akt were detected by western blotting after $24-\mathrm{h} 100 \mathrm{ng} / \mathrm{ml}$ TECK stimulation. (E and F) Cells were treated with the Akt inhibitor LY294002 or CCR9 siRNA before adding TECK. The expression levels of E-cadherin and Snail were detected by RT-qPCR after $24 \mathrm{~h} 100 \mathrm{ng} / \mathrm{ml}$ TECK stimulation. ${ }^{*} \mathrm{P}<0.05,{ }^{* *} \mathrm{P}<0.01,{ }^{* * *} \mathrm{P}<0.001$. TECK, thymus-expressed chemokine; EMT, epithelial-mesenchymal transition.

group vs. TECK group) or Akt phosphorylation inhibition (LY294002+TECK group vs. TECK group).

Overexpressing or inhibiting TECK expression can promote or inhibit EMT. The transfection efficiency of three different siRNAs was detected using western blotting, and it was revealed that siTECK-1 was the most effective at silencing TECK expression (Fig. 4A and B). Furthermore, the effect of siTECK was detected at the mRNA expression level (Fig. 4C and D) and similar effects were observed, thus siTECK-1 was used in follow-up experiments. The present results indicated that TECK expression, exogenous TECK or overexpressing TECK, contributed to increased EMT, marked with E-cadherin decrease and Snail increase compared to the control, while silenced TECK expression revealed a decrease of the EMT level (Fig. 4E and F).

TECK promotes cell growth by decreasing apoptosis instead of enhancing proliferation. During cell culture, it was revealed that TECK treatment contributed to increased cell proliferation. Moreover, the CCK-8 results (Fig. 5A and B) indicated that cell proliferation was increased compared to the control. Therefore, the present study examined the effect of TECK on cell proliferation and apoptosis in breast cancer. Cell cycle detection (Fig. 5C and D) results revealed that there was no difference in the cell cycle in the absence or presence of TECK. However, Hoechst 33342 staining (Fig. 5E) and AnnexinV/PI assay (Fig. 5F) results indicated that TECK could decrease the ratio of apoptotic cells induced by $\mathrm{H}_{2} \mathrm{O}_{2}$, and this change was disrupted by LY294002. Moreover, apoptosis-related protein markers were detected by western blotting, and similar results were obtained (Fig. 6A).

TECK expression affects the growth of breast cancer in vivo. For the tumor-bearing model experiment, there was no mice found dead over the course of the study and it was revealed that after 40 days the TECK overexpression xenograft volume was the largest, while the silenced TECK xenografts were the smallest (Fig. 6B and C). Furthermore, the same trend was identified with regards to the tumor weight (Fig. 6D; P<0.05). From the result of the relative tumor volume (Fig. 6E), it was observed that TECK was more effective in the MDA-MB-231 cell line than the MCF-7 cell line. 

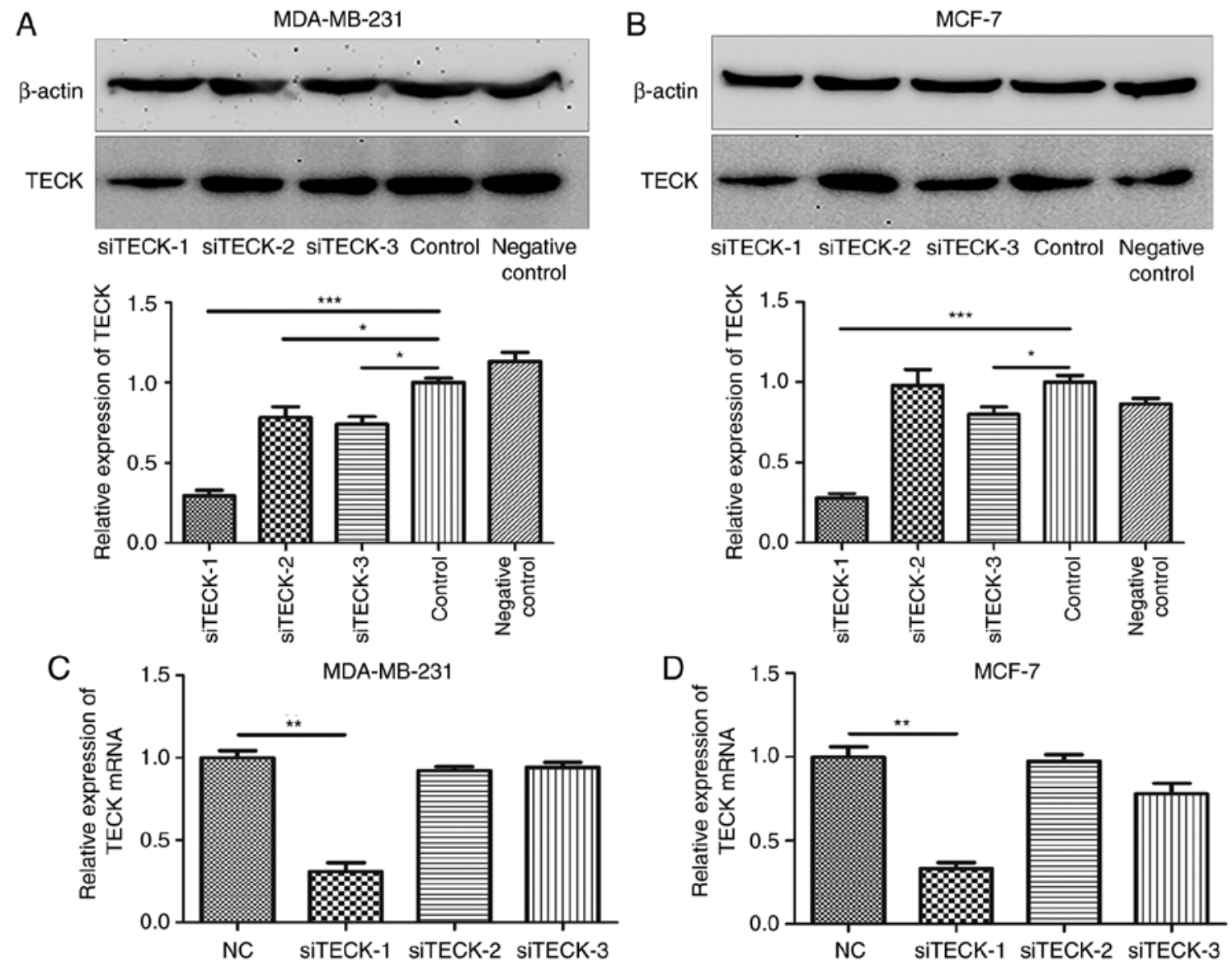

E

MDA-MB-231

F

MCF-7
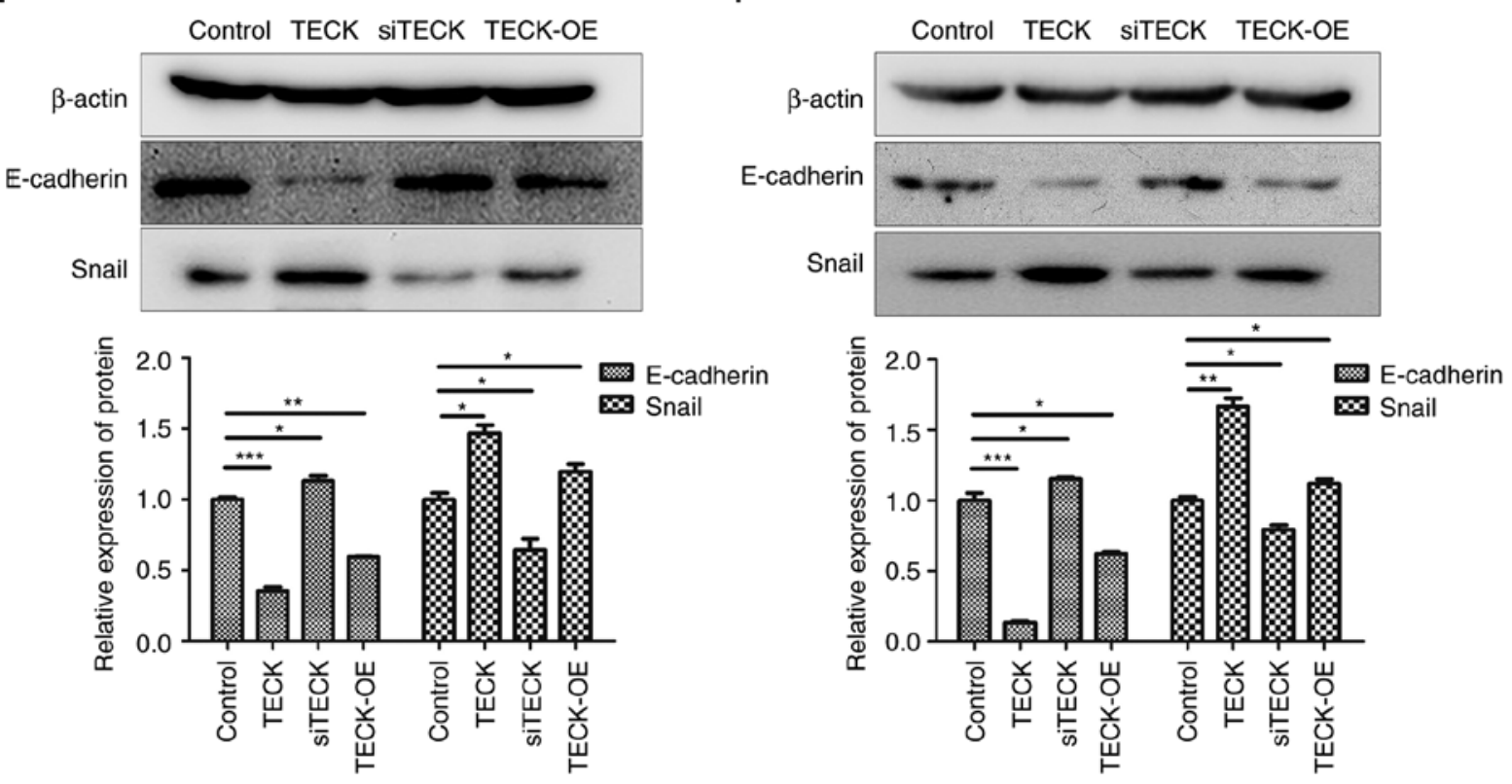

Figure 4. TECK silencing or overexpression can decrease or increase EMT levels in breast cancer cells. (A and B) Effect of three different TECK siRNAs was detected by western blotting. ${ }^{*} \mathrm{P}<0.05,{ }^{* *} \mathrm{P}<0.01,{ }^{* * *} \mathrm{P}<0.001$. (C and D) Effect of three different TECK siRNAs was detected by RT-qPCR. ${ }^{* *} \mathrm{P}<0.01$. (E) MDA-MB-231 and (F) MCF-7 cells were treated with $100 \mathrm{ng} / \mathrm{ml}$ TECK. The expression levels of E-cadherin and Snail in TECK stimulation, TECK silencing and TECK overexpression groups were detected by western blotting. ${ }^{*} \mathrm{P}<0.05,{ }^{* *} \mathrm{P}<0.01,{ }^{* * *} \mathrm{P}<0.001$. TECK, thymus-expressed chemokine; EMT, epithelial-mesenchymal transition.

\section{Discussion}

EMT is an important biological process in tumorigenesis and cancer progression, and during this process epithelial cells gain mesenchymal phenotypes (15), which is characterized by the reduction of an epithelial marker and increased mesenchymal markers. In the EMT process, epithelial cells lose cell polarity and cell-cell adhesion, and also acquire migratory and invasive 
A

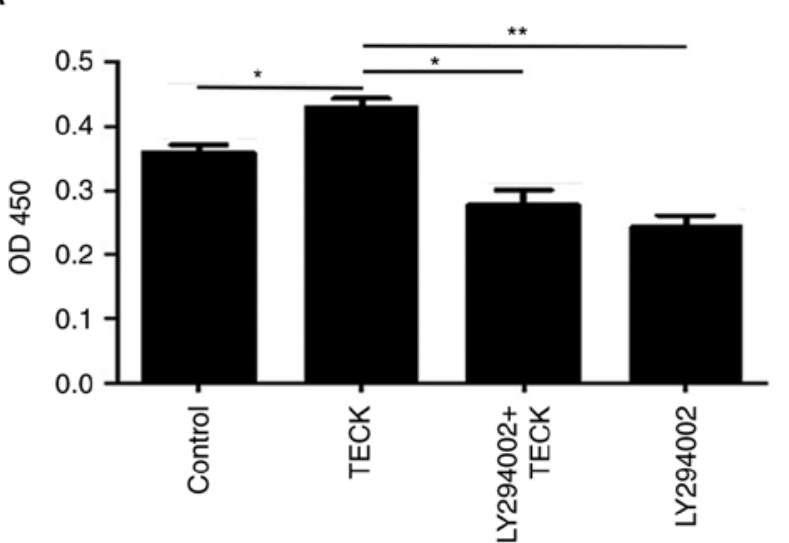

C

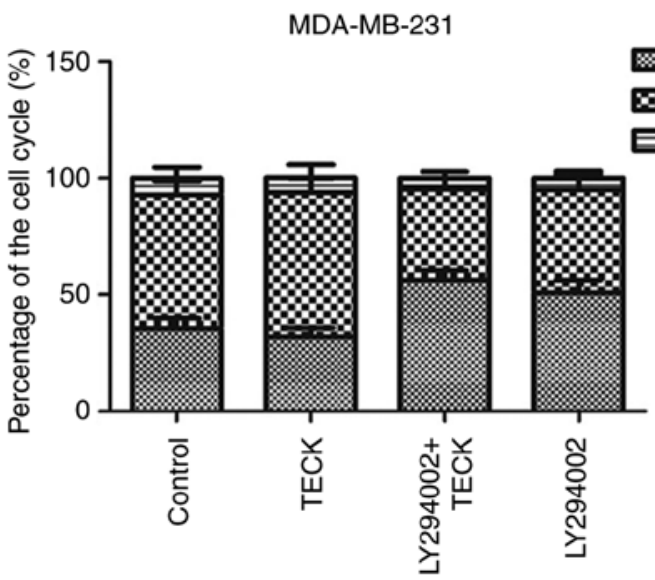

E

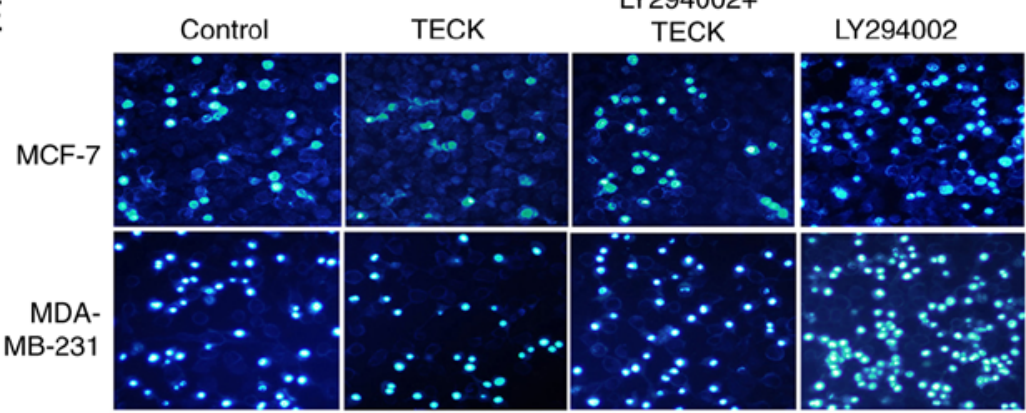

$\mathrm{F}$

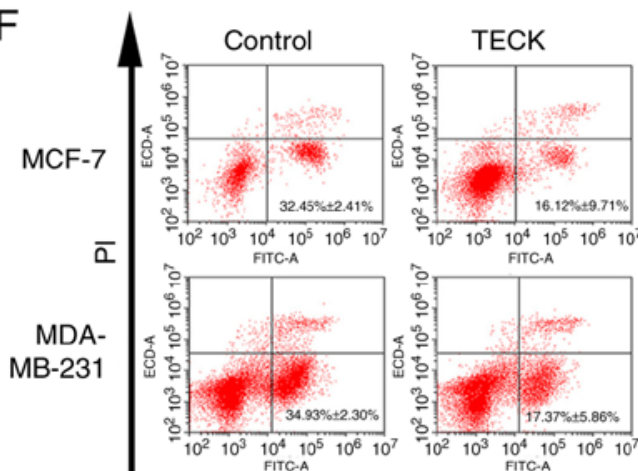

B

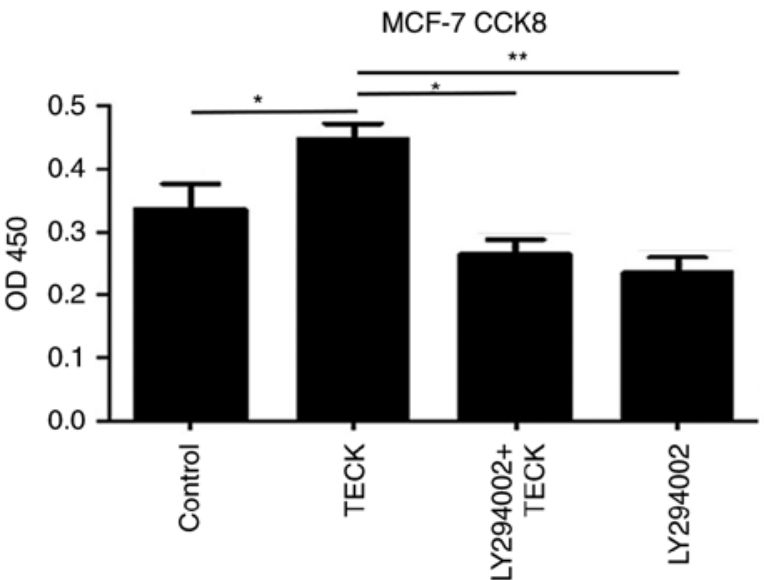

D

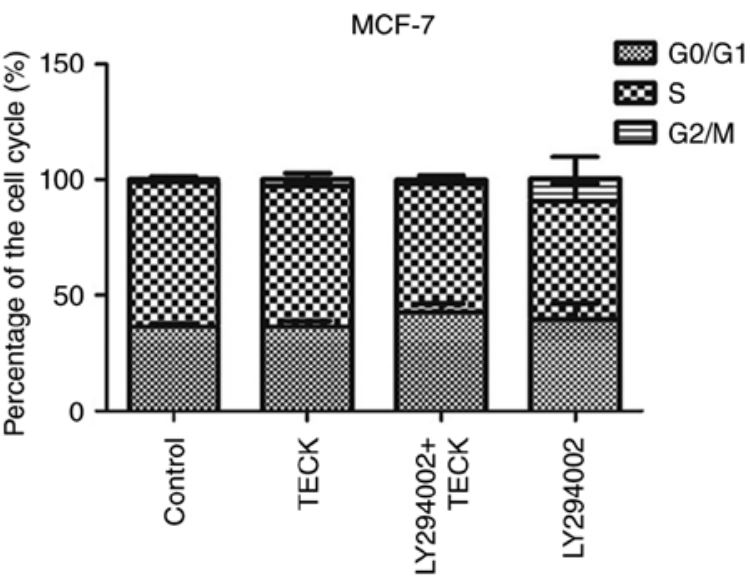

Annexin V-FITC

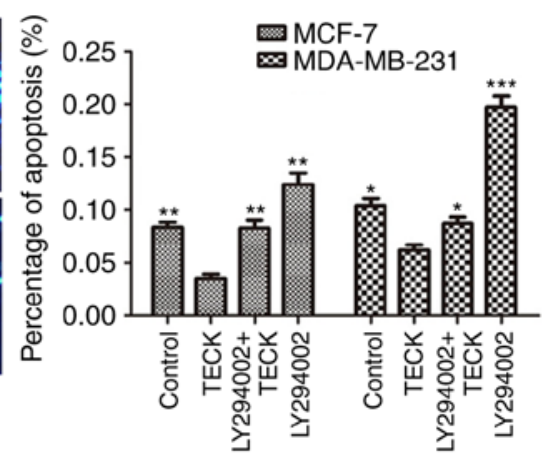

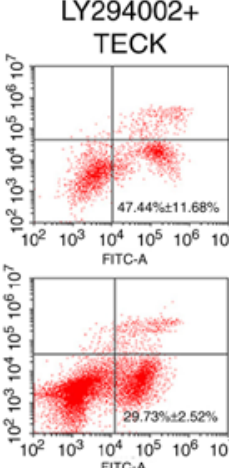

Y294002+

LY294002
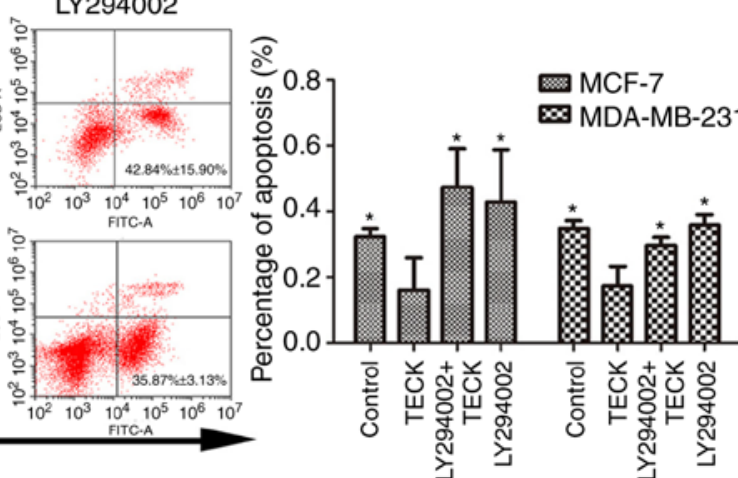

Figure 5. TECK stimulation increases the number of cells by inhibiting apoptosis, instead of influencing the cell cycle. (A) MDA-MB-231 and (B) MCF-7 cells were treated with/without LY294002 before $48 \mathrm{~h}$ TECK stimulation. The OD 450 was measured to determine the number of cells. ${ }^{*} \mathrm{P}<0.05$, ${ }^{* *} \mathrm{P}<0.01$. (C and D) Cells were treated with/without LY294002 before $48 \mathrm{~h}$ TECK stimulation, and stained with PI. Fluorescence was measured by flow cytometry and data were processed with ModFit. (E) MDA-MB-231 and MCF-7 cells were treated with blank, 100 ng/ml TECK, LY294002+100 ng/ml TECK and LY294002 for $24 \mathrm{~h}$, and then treated with $750 \mu \mathrm{M} \mathrm{H}_{2} \mathrm{O}_{2}$ to induce apoptosis. After $12 \mathrm{~h}$, the cells were stained with Hoechst $33342 .{ }^{*} \mathrm{P}<0.05,{ }^{* *} \mathrm{P}<0.01,{ }^{* * *} \mathrm{P}<0.001$. $(\mathrm{F}) \mathrm{Cells}$ were treated with/without LY294002 before 48 h TECK stimulation. Then, cells were stained using an Annexin V/PI kit and detected with flow cytometry. ${ }^{*} \mathrm{P}<0.05$. TECK, thymus-expressed chemokine. 

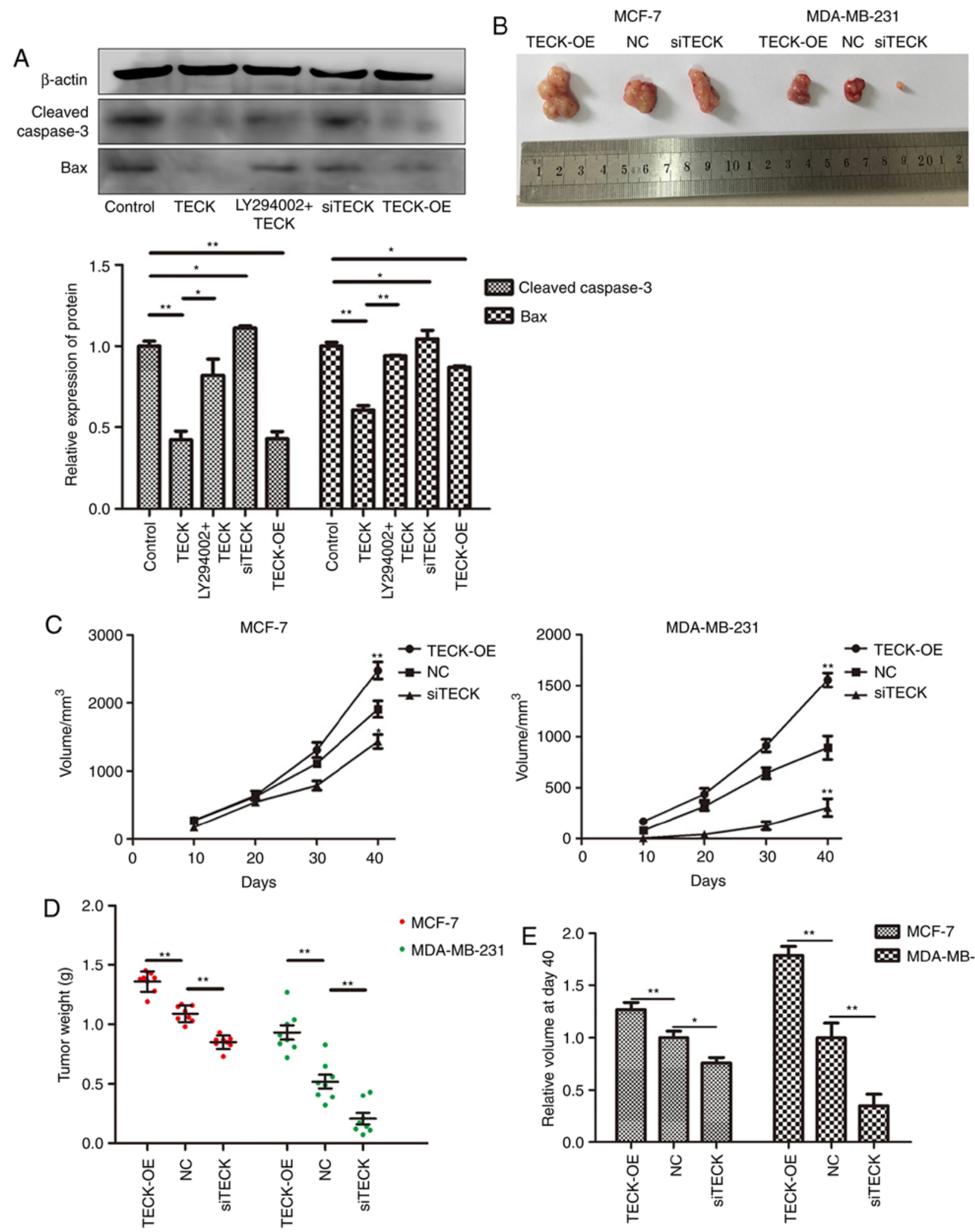

Figure 6. (A) Western blotting was used to detect cleaved caspase-3 and Bax protein expression levels to reflect apoptosis. ${ }^{*} \mathrm{P}<0.05,{ }^{* *} \mathrm{P}<0.01$. (B-D) TECK-OE, $\mathrm{NC}$ and siTECK cells (2 million) were injected into nude mice, then the tumor size was measured every 10 days and the tumor weight was weighed on the 40th day. ${ }^{*} \mathrm{P}<0.05,{ }^{* *} \mathrm{P}<0.01$. (E) The relative tumor volume compared to the NC group on the 40th day. TECK, thymus-expressed chemokine.

properties similar to mesenchymal stem cells (16). EMT can contribute to many cellular activities to enhance survival and improve the metastasis capacity of cancer cells. Moreover, previous studies have reported that EMT is associated with breast cancer stem cell development and multi-drug resistance (17), which are the primary reasons for failure of cancer treatment. Therefore, EMT is a target for clinical oncology therapy (18). The present results indicated that TECK could 


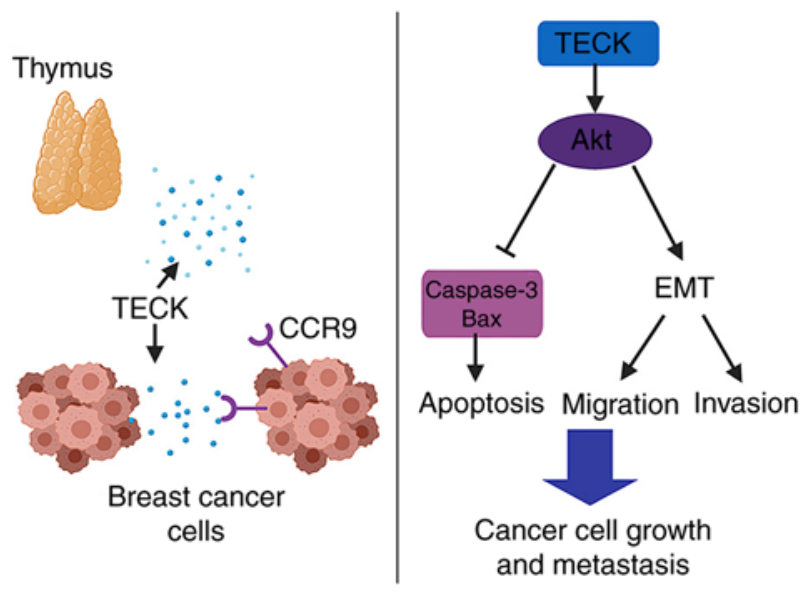

Figure 7. Proposed model of TECK-induced migration and invasion, and inhibition of apoptosis in human breast cancer cells. TECK, thymus-expressed chemokine.

promote the EMT process in a dose-dependent manner. The relationship between the capacity of the migration and invasion and EMT was also investigated, and it was revealed that TECK affected cell migration and invasion. Moreover, TECK could promote the EMT level in breast cancer and increase cell migration and invasion.

TECK has been widely studied in relation to the immune system and immune cells (19-22), but its effect in cancer cells requires further investigation. Heinrich et al (4) reported that pancreatic stellate cells can secrete TECK to activate pancreatic cancer cells. Furthermore, it has been revealed that serum TECK expression is elevated in patients with non-small cell lung cancer (23). However, it is not fully understood whether TECK can be secreted by cancer cells, and the role of TECK in cancer development remains unknown.

To the best of our knowledge, the present study is the first to demonstrate that breast cancer cells could secrete TECK in an autocrine manner, and that this secretion can promote breast cancer cell development. Thus, breast cancer cells may be activated by autocrine release of TECK.

As an endogenous immune substance, TECK can be secreted from thymus tissues, and a small amount from the small intestine and endometrial stromal cells. The results of IHC also demonstrated that an adult thymus secretes large amounts of TECK. The limitation of the present study is that a negative control was not set in the IHC experiments although we all know that thymus tissues can secrete TECK. TECK is important for immune cell development, but can also promote carcinogenesis and progression in cancers (24). However, breast cancer cells can secrete TECK themselves, and TECK expression may be associated with survival rate (11). Therefore, it is important to investigate the effect of TECK in breast cancer.

The present results indicated that TECK could play a role in breast cancer progression, the EMT process, and cell migration, invasion and apoptosis, which were mediated by the Akt signaling pathway. Thymus tissues are replaced by adipose tissues in adults, but thymus tissues can still secrete abundant levels of TECK. Furthermore, TECK can be transported to the cancer site via the blood flow, which acts as a stimulation factor to breast cancer cells.
Metastasis and drug-resistance are the main reasons for the failure of treatment for breast cancer. A previous study revealed that TECK was associated with breast cancer metastasis and drug-resistance (12), and it could also regulate immune cell functions around the cancer cells. Common metastatic sites include the lymph nodes, lung, bone and brain, however small intestine and thymus metastases are often identified in patients with breast cancer, with a reported rate of 16 and $11 \%$, respectively (25), but the mechanism of this is not fully understood.

Studies have shown that chemokines cause the transfer of cancer cells to specific organs in the same way as causing movement and displacement of leukocytes given their chemotaxis properties $(26,27)$. TECK, which is also a chemokine, may have a potential role in the process of breast cancer cell metastasis to specific organs, which is thought to be similar to the mechanism of leukocytes chemotaxis. Thus, it is speculated that TECK may be a key substance in the process of breast cancer cell metastasis to the thymus and small intestine, where a high expression level of TECK was identified, which may be related to its strong chemotaxis ability.

During carcinogenesis and the progression of breast cancer, the immune organ, thymus and its secretory protein TECK may act as helpers, despite the primary function of the immune system to attack cancer cells.

The present results revealed that TECK could regulate several cellular functions in breast cancer cells via the Akt signaling pathway (Fig. 7). The present results may facilitate the development of novel therapeutic strategies to treat breast cancer. It may be possible to develop new drugs to block TECK secretion or function, or inhibit the phosphorylation of Akt, which cannot only reduce the level of EMT, but also inhibit cell migration and invasion in breast cancer.

Currently, there are few effective therapies to treat triple-negative breast cancer (TNBC), and the present results indicated that TECK was most effective in the TNBC cell line, particularly in the apoptosis assay and xenograft model. Therefore, the present results could provide a basis for effective treatments for patients with TNBC. Moreover, inhibition of TECK may be a key target to prevent breast cancer specific organ-oriented metastasis to the small intestine and thymus.

\section{Acknowledgements}

Not applicable.

\section{Funding}

No funding was received.

\section{Availability of data and materials}

The datasets used and/or analyzed during the present study are available from the corresponding author on reasonable request.

\section{Authors' contributions}

LC, YS and LQ performed the experiments. ZZo and HT made substantial contributions to the concept and design of the present study. SZ and ZZa conducted data analysis and 
interpretation of the results. LC and SZ wrote the manuscript. All authors read, revised and approved the manuscript and agreed to be accountable for all aspects of the research in ensuring that the accuracy or integrity of any part of the work are appropriately investigated and resolved.

\section{Ethics approval and consent to participate}

This study was approved by the Ethics committees of Shandong Provincial Hospital (NSFC: approval no. 2019-201). Non-retrospective ethical approval for the animal experiments conducted in the study were obtained by the Use Committee for Animal Care of Shandong University. In addition, it is confirmed that the tumor burden did not exceed the recommended dimensions and that animals were anesthetized and sacrificed using acceptable methods/techniques. Informed consent was obtained before using thymus tissue paraffin sections.

\section{Patient consent to participate}

Not applicable.

\section{Competing interests}

The authors declare that they have no competing interests.

\section{References}

1. Siegel RL, Miller KD and Jemal A: Cancer statistics, 2019. CA Cancer J Clin 69: 7-34, 2019.

2. Fan L, Strasser-Weippl K, Li JJ, St Louis J, Finkelstein DM, Yu KD, Chen WQ, Shao ZM and Goss PE: Breast cancer in China. Lancet Oncol 15: e279-e289, 2014.

3. DeSantis CE, Fedewa SA, Goding Sauer A, Kramer JL, Smith RA and Jemal A: Breast cancer statistics, 2015: Convergence of incidence rates between black and white women. CA Cancer J Clin 66: 31-42, 2016.

4. DeSantis CE, Ma J, Gaudet MM, Newman LA, Miller KD, Goding Sauer A, Jemal A and Siegel RL: Breast cancer statistics, 2019. CA Cancer J Clin 69: 438-451, 2019.

5. Marcuzzi E, Angioni R, Molon B and Calì B: Chemokines and chemokine receptors: Orchestrating tumor metastasization. Int J Mol Sci 20: E96, 2018.

6. Franciszkiewicz K, Boissonnas A, Boutet $\mathrm{M}$, Combadière $\mathrm{C}$ and Mami-Chouaib F: Role of chemokines and chemokine receptors in shaping the effector phase of the antitumor immune response. Cancer Res 72: 6325-6332, 2012.

7. Svensson M and Agace WW: Role of CCL25/CCR9 in immune homeostasis and disease. Expert Rev Clin Immunol 2: 759-773, 2006.

8. Heinrich EL, Arrington AK, Ko ME, Luu C, Lee W, Lu J and Kim J: Paracrine activation of chemokine receptor CCR9 enhances the invasiveness of pancreatic cancer cells. Cancer Microenviron 6: 241-245, 2013.

9. Li MQ, Wang Y, Chang KK, Meng YH, Liu LB, Mei J, Wang Y, Wang XQ, Jin LP and Li DJ: CD4+Foxp3+ regulatory T cell differentiation mediated by endometrial stromal cell-derived TECK promotes the growth and invasion of endometriotic lesions. Cell Death Dis 5: e1436, 2014.
10. Vicari AP, Figueroa DJ, Hedrick JA, Foster JS, Singh KP, Menon S, Copeland NG, Gilbert DJ, Jenkins NA, Bacon KB and Zlotnik A: TECK: A novel CC chemokine specifically expressed by thymic dendritic cells and potentially involved in T cell development. Immunity 7: 291-301, 1997.

11. Thomas JK, Mir H, Kapur N, Bae S and Singh S: CC chemokines are differentially expressed in breast cancer and are associated with disparity in overall survival. Sci Rep 9: 4014, 2019.

12. Wang C, Liu Z, Xu Z, Wu X, Zhang D, Zhang Z and Wei J: The role of chemokine receptor 9/chemokine ligand 25 signaling: From immune cells to cancer cells. Oncol Lett 16: 2071-2077, 2018.

13. Livak KJ and Schmittgen TD: Analysis of relative gene expression data using real-time quantitative PCR and the 2(-Delta Delta C(T)) method. Methods 25: 402-408, 2001.

14. Wang X, Liu R, Zhu W, Chu H, Yu H, Wei P, Wu X, Zhu H, Gao H, Liang J, et al: UDP-glucose accelerates SNAI1 mRNA decay and impairs lung cancer metastasis. Nature 571: 127-131, 2019.

15. Greenburg G and Hay ED: Epithelia suspended in collagen gels can lose polarity and express characteristics of migrating mesenchymal cells. J Cell Biol 95: 333-339, 1982.

16. Tomaskovic-Crook E, Thompson EW and Thiery JP: Epithelial to mesenchymal transition and breast cancer. Breast Cancer Res 11: 213, 2009.

17. Mallini P, Lennard T, Kirby J and Meeson A: Epithelial-tomesenchymal transition: What is the impact on breast cancer stem cells and drug resistance. Cancer Treat Rev 40: 341-348, 2014.

18. Santamaria PG, Moreno-Bueno G, Portillo F and Cano A: EMT: Present and future in clinical oncology. Mol Oncol 11: 718-738, 2017.

19. Zhou C, Wu J, Borillo J, Torres L, McMahon J and Lou YH: Potential roles of a special CD8 alpha alpha + cell population and $\mathrm{CC}$ chemokine thymus-expressed chemokine in ovulation related inflammation. J Immunol 182: 596-603, 2019.

20. Meurens F, Whale J, Brownlie R, Dybvig T, Thompson DR and Gerdts V: Expression of mucosal chemokines TECK/CCL25 and MEC/CCL28 during fetal development of the ovine mucosal immune system. Immunology 120: 544-555, 2007.

21. Zabel BA, Agace WW, Campbell JJ, Heath HM, Parent D, Roberts AI, Ebert EC, Kassam N, Qin S, Zovko M, et al: Human G protein-coupled receptor GPR-9-6/CC chemokine receptor 9 is selectively expressed on intestinal homing $\mathrm{T}$ lymphocytes, mucosal lymphocytes, and thymocytes and is required for thymus-expressed chemokine-mediated chemotaxis. J Exp Med 190: 1241-1256, 1999.

22. Kunkel EJ, Campbell JJ, Haraldsen G, Pan J, Boisvert J, Roberts AI, Ebert EC, Vierra MA, Goodman SB, Genovese MC, et al: Lymphocyte CC chemokine receptor 9 and epithelial thymus-expressed chemokine (TECK) expression distinguish the small intestinal immune compartment: Epithelial expression of tissue-specific chemokines as an organizing principle in regional immunity. J Exp Med 192: 761-768, 2000.

23. Gupta P, Sharma PK, Mir H, Singh R, Singh N, Kloecker GH, Lillard JW Jr and Singh S: CCR9/TECK expression in non-small cell lung cancer correlates with aggressive disease and mediates key steps of metastasis. Oncotarget 30: 10170-10179, 2014.

24. Tu Z, Xiao R, Xiong J, Tembo KM, Deng X, Xiong M, Liu P, Wang $M$ and Zhang Q: CCR9 in cancer: Oncogenic role and therapeutic targeting. J Hematol Oncol 16: 10, 2016.

25. Cifuentes $\mathrm{N}$ and Pickren JW: Metastases from carcinoma of mammary gland: An autopsy study. J Surg Oncol 11: 193-205, 1979.

26. Rezaeeyan H, Shirzad R, McKee TD and Saki N: Role of chemokines in metastatic niche: New insights along with a diagnostic and prognostic approach. APMIS 126: 359-370, 2018.

27. Ben-Baruch A: Organ selectivity in metastasis: Regulation by chemokines and their receptors. Clin Exp Metas 25: 345-356, 2008 .

This work is licensed under a Creative Commons Attribution-NonCommercial-NoDerivatives 4.0 International (CC BY-NC-ND 4.0) License. 